\title{
O direito à recusa de tratamento e o papiloma escamoso oral: Relato de caso
}

\author{
The right to refuse treatment and oral scammus papilloma: Case report \\ EI derecho a rechazar el tratamiento y el papiloma de scammus oral: Reporte de caso
}

Recebido: 18/04/2021 | Revisado: 24/04/2021 | Aceito: 27/04/2021 | Publicado: 12/05/2021

Anna Danielly Almeida do Nascimento

ORCID: https://orcid.org/0000-0002-4424-1039

Universidade de Pernambuco, Brasil

E-mail: annadanielly1@gmail.com

Beatriz Santos Borges

ORCID: https://orcid.org/0000-0002-9787-5932

Universidade de Pernambuco, Brasil

E-mail: beatrizsborgess@gmail.com

Fernanda Teles Pereira

ORCID: https://orcid.org/0000-0002-7802-8930 Universidade de Pernambuco, Brasil

E-mail: fernandatelespereira@gmail.com

Cristianne de Barros Santos

ORCID: https://orcid.org/0000-0001-7430-9025

Universidade de Pernambuco, Brasil

E-mail: crisbarros18@hotmail.com

Angélica Lopes Frade

ORCID: https://orcid.org/0000-0002-0110-8043

Universidade de Pernambuco, Brasil

E-mail: angélica.frade@outlook.com

Laís Azevedo Lins de Holanda

ORCID: https://orcid.org/0000-0001-8967-018X

Universidade Federal de Pernambuco, Brasil E-mail: laisalholanda@hotmail.com

Mayara Larissa Moura de Souza

ORCID: https://orcid.org/0000-0001-7023-7829

Universidade Federal de Pernambuco, Brasil

E-mail: mayara.mourasouza@gmail.com

Isabela Vicência Menezes Castelo Branco ORCID: https://orcid.org/0000-0001-6269-1570

Universidade Federal de Pernambuco, Brasil E-mail: isabelamcbranco@gmail.com

Maria Gabriela Cavalcanti de Araújo ORCID: https://orcid.org/0000-0003-3049-6118 Universidade de Pernambuco, Brasil E-mail: gabryellapardo@hotmail.com

Juliana Cavalcante de Oliveira ORCID: https://orcid.org/0000-0001-5684-3443

Universidade Federal de Pernambuco, Brasil

E-mail: julianacavalcante_@hotmail.com

Maria Cecília Freire de Melo

ORCID: https://orcid.org/0000-0002-9592-4796

Universidade Federal de Pernambuco, Brasil E-mail: mceciliafreire@ hotmail.com

Thuanny Silva de Macedo

ORCID: https://orcid.org/0000-0003-0036-3971

Universidade Federal de Pernambuco, Brasil

E-mail: thuannymacedo16@gmail.com

Fátima Cristina Mendes Matos

ORCID: https://orcid.org/0000-0002-9135-8117 Universidade de Pernambuco, Brasil

E-mail: fcmmatos@hotmail.com

Aurora Karla de Lacerda Vidal

ORCID: https://orcid.org/0000-0003-2831-2471 Universidade de Pernambuco, Brasil

E-mail: aurora.vidal@upe.br 


\begin{abstract}
Resumo
O Papiloma Escamoso oral é uma neoplasia benigna de origem epitelial, bastante comum, associado à infecção pelo Papiloma Vírus Humano (HPV). Clinicamente apresenta-se como nódulo exofítico, mole, indolor e geralmente pedunculado, com aspecto verrucoso/papilomatoso. Esse trabalho tem por objetivo relatar um caso de uma paciente com diagnóstico clínico e histopatológico de papiloma escamoso oral de grande dimensão, com focos de displasia epitelial moderada, localizado em mucosa jugal e face interna de lábios do lado direito. O tratamento inicialmente indicado foi a excisão cirúrgica e, posteriormente a excisão cirúrgica com reconstrução plástica, porém a paciente em decorrência de questões sociais, medo e ausência de apoio familiar, mesmo sabendo dos riscos de transformação maligna da lesão recusou o tratamento. Apesar da necessidade de tratamento a paciente possui autonomia para tomada de decisões e o direito à recusa de tratamento é reconhecido pelo Código Civil brasileiro como um dos direitos da personalidade. Respeitar a autonomia da paciente caracteriza um sistema de saúde humanizado e constitui um dos principais desafios enfrentados pelos profissionais de saúde.
\end{abstract}

Palavras-chave: Papiloma; Cirurgia bucal; Autonomia pessoal; Princípios morais; Bioética.

\begin{abstract}
Oral squamous papilloma is a benign neoplasm of epithelial origin, quite common, associated with infection by the Human Papilloma Virus (HPV). Clinically, it presents as an exophytic nodule, soft, painless and generally pedunculated, with a verrucous / papillomatous aspect. This study aims to report a case of a patient with a clinical and histopathological diagnosis of large oral squamous papilloma, with foci of moderate epithelial dysplasia, located in the cheek mucosa and inner side of the lips on the right side. The treatment initially indicated was surgical excision and, later, surgical excision with plastic reconstruction, but the patient due to social issues, fear and lack of family support, even knowing the risks of malignant transformation of the lesion, refused treatment. Despite the need for treatment, the patient has autonomy to make decisions and the right to refuse treatment is recognized by the Brazilian Civil Code as one of the rights of the personality. Respecting the patient's autonomy characterizes a humanized health system and constitutes one of the main challenges faced by health professionals.
\end{abstract}

Keywords: Papilloma; Surgery oral; Personal autonomy; Morals; Bioethics.

\title{
Resumen
}

El papiloma escamoso oral es una neoplasia benigna de origen epitelial, bastante común, asociada a la infección por el virus del papiloma humano (VPH). Clínicamente se presenta como un nódulo exofítico, blando, indoloro y generalmente pediculado, de aspecto verrugoso / papilomatoso. Este estudio tiene como objetivo reportar el caso de un paciente con diagnóstico clínico e histopatológico de papiloma escamoso oral de gran tamaño, con focos de displasia epitelial moderada, localizados en la mucosa de la mejilla y cara interna de los labios del lado derecho. El tratamiento inicialmente indicado fue la escisión quirúrgica y, posteriormente, la escisión quirúrgica con reconstrucción plástica, pero el paciente por cuestiones sociales, miedo y falta de apoyo familiar, aun conociendo los riesgos de transformación maligna de la lesión, rechazó el tratamiento. A pesar de la necesidad de tratamiento, el paciente tiene autonomía para tomar decisiones y el derecho a rechazar el tratamiento está reconocido por el Código Civil brasileño como uno de los derechos de la personalidad. El respeto a la autonomía del paciente caracteriza a un sistema de salud humanizado y constituye uno de los principales desafíos que enfrentan los profesionales de la salud.

Palabras clave: Papiloma; Cirugía bucal; Autonomía personal; Principios morales; Bioética.

\section{Introdução}

O tema "ética" reconquistou o seu espaço nas discussões internacionais, nas últimas décadas do século XX, e ocorreu um súbito interesse pela chamada ética prática ou aplicada, surgindo três novas áreas de estudo: a ética ambiental, a ética dos negócios e a bioética. Esta se refere a uma disciplina que estuda a ética das situações de vida, abrangendo uma lista extensa de problemas que se relacionam com a saúde, referindo-se aos direitos e obrigações dos profissionais, dos pacientes e da sociedade e sobre esta será relatado um caso clínico (Ramos, 2009; Ramos \& Junqueira, 2007; Guimarães, 2013; Singer, 1994; Kottow, 1995). Dessas diferentes orientações da bioética surgiram duas perspectivas: a anglo-americana, denominada perspectiva principialista, mais individualista, uma vez que privilegia a autonomia da pessoa singular (microproblemas); e a europeia, conhecida como perspectiva personalista e humanista, a qual concentra mais fortemente a sua atenção nos interesses morais mais coletivos (macroproblemas) com uma preocupação acentuada pela dimensão social do homem, colocando maior ênfase no sentido de justiça (Carvalho Fortes \& Pavone Zoboli, 2009). 
No Brasil, antes da Constituição de 1988 a ideia de saúde era apenas uma noção de ausência de doença, mas após a constituição esse entendimento modificou-se. A Organização Mundial de Saúde, já em 1946, defendia a ideia de saúde como "um estado de completo bem-estar físico, mental e social e não apenas a ausência de distúrbios e doenças". A abrangência do conceito de saúde passou a requerer uma mudança na organização e prestação desse serviço público, na medida em que, além das causas biológicas da doença, também passaram a ser consideradas as causas sociais tais como a fome e, a ausência de saneamento básico, a ausência de moradia, a ausência de cultura, a ausência de empregos, o combate à violência, a defesa do meio ambiente e, melhor distribuição de renda, enfim, todas as condições necessárias a uma vida saudável e produtiva para os seres humanos (Paulino et al., 2009; WHO, 2001).

Uma grande parcela da população brasileira não tem condições financeiras de pagar um plano de saúde e recorre ao Sistema Único de Saúde - SUS, que está disciplinado pela Lei 8.080/90. Dentre seus princípios e diretrizes norteadores estão a universalidade de acesso aos serviços de saúde em todos os níveis de assistência; integralidade de assistência, entendida como um conjunto articulado e contínuo das ações e serviços preventivos e curativos, individuais e coletivos, exigidos para cada caso em todos os níveis de complexidade do sistema; preservação da autonomia das pessoas na defesa de sua integralidade física e moral; igualdade da assistência à saúde sem preconceitos ou privilégios de qualquer espécie; direito à informação, às pessoas assistidas, sobre sua saúde e etc (Brasil, 2004; Brasil, 2008) . As grandes mudanças na Lei 8.080 foram acompanhadas por um conjunto de Normas Operacionais na década de 90 e, desde então, o SUS vem sendo gradativamente implantado e aprimorado em todo território nacional (Brasil, 2004; Brasil, 2008; Bourget, 2006; Nickel et al., 2008).

Deste modo, considerando a infecção da mucosa oral pelo Papiloma Vírus Humano (HPV), o qual pode ocorrer através do sexo oral, por contato direto com objetos contaminados, autoinoculação e, inclusive, transmissão via placenta ou leite materno (Ferraro et al., 2011; Grm et al., 2009). Acredita-se que o HPV infecte epitélios por meio de abrasões ou microlacerações da pele e da mucosa, por exemplo, durante o intercurso sexual, permitindo o acesso do vírus às células da camada basal. Os vírus lesam as células do hospedeiro ao entrarem nestas, e se replicam as suas custas (Pereira et al., 2007; Feller et al., 2009).

Dos mais de 200 tipos de HPV identificados, pelo menos 25 têm sido detectados em lesões orais. Os mais comumente encontrados são os tipos 6 e 11, associados com as lesões benignas, e os tipos 16 e 18 comprovadamente carcinogênicos e possivelmente associados aos carcinomas orais (Vidal et al., 2004; Vidal et al., 2006; Da Silva et al., 2016; Simonato \& Miyahara, 2007). As manifestações orais associadas ao HPV são descritas clinicamente como lesões assintomáticas, com áreas brancas puntiformes ou extensas, podem ser pediculadas ou sésseis e a superfície pode variar de finamente granular à papilar, e são diagnosticadas histopatologicamente como: papiloma escamoso, condiloma acuminado, verruga vulgar ou hiperplasia epitelial focal, que são as variantes benignas do epitélio escamoso estratificado. Havendo, ainda, a associação a quadros de leucoplasia, líquen plano e carcinoma de células escamosas (Ferraro et al., 2011; Castro et al., 2004; Vidal et al., 2006).

Além de ser um fator de risco carcinogênico, a infecção da mucosa pelo HPV pode aumentar a susceptibilidade do epitélio, sendo capaz de intensificar ou alterar a ação carcinogênica do álcool e do tabaco passando a potencializar o desenvolvimento do câncer de boca e orofaringe, sendo considerado também um fator co-carcinogênico (Vidal et al., 2004; Vidal et al., 2006; Montenegro et al., 2014).

O sucesso no tratamento de lesões orais causadas pelo HPV depende de uma boa comunicação entre profissional e paciente, além de uma boa aceitação e colaboração do paciente ao plano de tratamento indicado, que pode ser cirúrgico ou medicamentoso. A excisão cirúrgica conservadora, incluindo a base da lesão, é o tratamento adequado para o papiloma escamoso oral. A recidiva é improvável e quando as lesões são tratadas em estágios iniciais o prognóstico se torna melhor. Os fatores que podem influenciar na escolha do tratamento são: tamanho, número e local da lesão, além de sua morfologia, conveniência, efeitos adversos e a experiência do profissional da saúde diante de tal lesão (Cardoso, 2012). 
Esta comunicação profissional-paciente é essencial para a realização da anamnese e para transmissão de dados referentes ao diagnóstico, tratamento e prognóstico do indivíduo em questão, e resguarda, desta forma, a obrigação ética do profissional. O Código de Ética Odontológico brasileiro, refere que o Cirurgião-Dentista é obrigado a informar ao paciente o diagnóstico, esclarecer adequadamente os propósitos, riscos, custos e alternativas do tratamento (CFO, 2012). A bioética mostra que a comunicação é a melhor forma de prevenção e dissolução de problemas e não pode ser relegada pelo profissional, pois permite a análise e ponderação de conflito de interesses e valores que surgem no encontro entre o profissional e o paciente/familiar, a fim de resolver dilemas morais advindos do campo da assistência em saúde.

As percepções dos pacientes durante a revelação de diagnósticos, prognósticos e planos de tratamento podem ser influenciadas por fatores pessoais, sociodemográficos e conceitos culturalmente construídos, como o princípio da autonomia do paciente e o papel da família. Essas particularidades podem nortear a forma e o tipo de comunicação e informação a ser fornecida, e até mesmo o tipo de suporte emocional ofertado (Porensky \& Carpenter, 2016).

Segundo Garrafa (1995), a bioética é uma ferramenta teórica e metodológica entendida como a resultante moral do conjunto de decisões, políticas e sanitárias, individuais e coletivas, que proporcionam aumento da cidadania e diminuição da exclusão social. A comunicação e a bioética são coadjuvantes da prática médica e são inseparáveis da prática clínica (Schramm, 2001).

Beauchamp e Childress (2002) publicaram, em 1979, os princípios da bioética, que são: autonomia, beneficência, não maleficência e justiça, sendo considerados como o fundamento da reflexão ética e sua aplicação apresenta resultados positivos a respeito da dignidade humana. O princípio da autonomia, diz respeito à liberdade de opinião e decisão do paciente, e privacidade de ação, ou seja, agir de acordo com a própria intenção (Beauchamp \& Childress, 2002). No entanto, segundo Ramos e Junqueira (2007), a autonomia está em segundo plano, pois se for para o bem da pessoa, até que ponto ela poderá decidir o que é melhor para si, se não entende de medicina, odontologia ou de outra área específica de conhecimento?

Assim, neste artigo segue relatado um caso clínico de um indivíduo com histórico de mordedura crônica em mucosa jugal direita e portador de papiloma escamoso oral, o qual, em virtude de questões pessoais, familiares e sociais, recusou o tratamento cirúrgico indicado. Sabe-se que na ausência de tratamento, a progressão dessa lesão papilomatosa poderá implicar em comprometimento clínico (estético/funcional) e progressão histopatológica caracterizada por atipias celulares displásicas e neoplásicas, tendo em vista que o vírus HPV pode comportar-se como mais um co-carcinógeno para o câncer de boca, à semelhança do carcinoma uterino (Vidal et al., 2004; Vidal et al., 2006).

A liberdade é um bem moral que precisa ser defendido, mas o ser humano é um ser social, fruto de relações familiares e dependente de vínculos sociais. Essas relações determinam limites às liberdades individuais e impõem responsabilidades diante das consequências dos atos individuais na vida dos outros. Os vínculos fortalecem, a independência fragiliza. Não se pode falar de "liberdade" sem considerar a "responsabilidade" dos atos. Na lógica individualista, esse princípio é absoluto. Contudo, o princípio ético da autonomia é empregado em seu verdadeiro valor quando implica o reconhecimento de que cada pessoa humana merece ser respeitada nas suas opiniões, como poderá ser verificado no caso descrito a seguir.

\section{Metodologia}

Respeitando-se os princípios de autonomia e as normas para pesquisa em seres humanos, este estudo integra projeto de pesquisa submetido ao Comitê de Ética em Pesquisa da Universidade de Pernambuco e aprovado sob o parecer $\mathrm{n}^{\circ}$. 3.184.856. Trata-se de um relato de caso original, primário, contendo características importantes acerca do processo patológico, procedimentos realizados e sobre a participante, a qual assinou o Termo de Consentimento Livre e Esclarecido, consentindo a divulgação de seu caso para fins acadêmicos.

Consoante a literatura (Albrecht et al., 2005; Bardin, 2010; Estrela, 2018; Pereira et al., 2018; Koche, 2011; Lakatos 
\& Marconi, 2003) um caso para ser relatado, investigado, analisado e discutido precisa ter alguma particularidade que o diferencie, tem que ser especial. Um bom relato de caso deve ter o objetivo de acrescentar benefícios às práticas atuais ou de traçar possíveis novas direções na pesquisa de determinado tema em que um único ou poucos indivíduos possam ser representativos, destacam Albrecht et al., (2005), tal qual segue descrito neste cuidadoso e detalhado relato de um caso clínico.

Quanto aos possíveis riscos e desconfortos, de acordo com a Resolução 466/2012, de 12 de dezembro de 2012, sobre as Normas Regulamentadoras de Pesquisas envolvendo seres humanos, de origem física, psíquica, moral, intelectual, social, cultural ou espiritual, eles foram contornados e controlados através da valorização, preservação e sigilo das informações e privacidade da participante.

Os benefícios esperados com o resultado desse relato é permitir uma melhor compreensão tanto do processo biológico quanto da bioética, os quais regem a prática médica-odontológica. Consoante Yin (2015) os métodos qualitativos e quantitativos de investigação não se excluem e podem ser importantes se complementando quali-quantitativamente e permitindo um melhor entendimento dos fenômenos em estudo, reforçou Bardin (2010).

\section{Relato de Caso}

Gênero feminino, 61 anos, viúva, negra, com manifestações de vitiligo e perda auditiva parcial, natural de Jaboatão dos Guararapes, Pernambuco, Nordeste, Brasil. Assinou o Termo de Consentimento Livre e Esclarecido, consentindo a divulgação de seu caso para fins acadêmicos, estudo aprovado sob o parecer $n^{\circ}$. 3.184.856 HUOC/UPE. Foi encaminhada por cirurgiã-dentista da Atenção Básica em Saúde, da Cidade do Recife, para o Serviço de Odontologia do Centro de Oncologia do Hospital Universitário Oswaldo Cruz (CEON/HUOC/UPE) com finalidade de diagnóstico de lesão oral. Ao exame físico intraoral foi observado indivíduo parcialmente dentado, em uso de próteses dentárias superior e inferior mal adaptadas, hábito de mordedura constante da mucosa jugal direita, apresentando lesão indolor, papilomatosa/verrucosa, medindo aproximadamente $6 \mathrm{~cm}$, comprometendo a mucosa jugal direita e mucosa labial superior e inferior; além de lesão leucoeritroplásica em mucosa jugal esquerda (Figura 1).

Figura 1. Imagem inicial. Aspecto clínico de lesão papilomatosa em mucosa jugal direita (2016).

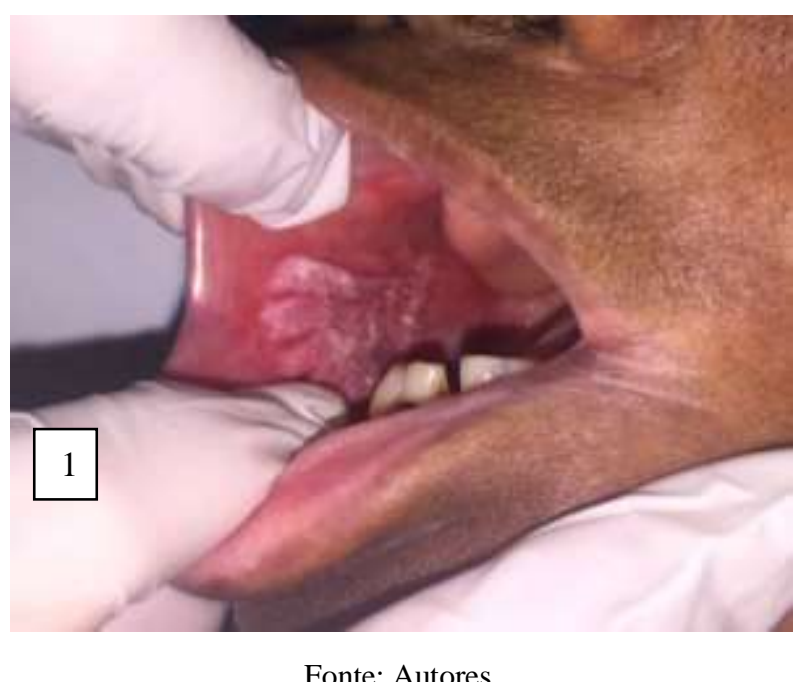

Ambas as lesões orais sugestivas de traumas por mordedura. Ao exame extraoral não foram encontradas anormalidades dignas de nota. Foi realizada a biópsia incisional da lesão situada em mucosa jugal direita, sob anestesia local, sem intercorrências, em novembro de 2016, para a conclusão diagnóstica. O espécime coletado foi encaminhado para análise, 
sendo o laudo anatomopatológico conclusivo para Papiloma Escamoso Oral, sugestivo de infecção por HPV (AP.165066). Após a entrega do laudo, explicação do processo patológico e indicação de tratamento a paciente sumiu, não retornou às consultas subsequentes, reaparecendo em fevereiro de 2017. Persistindo a suspeita clínica de malignidade foi realizada nova biópsia, em 10/02/2017, cujo laudo anatomopatológico manteve o diagnóstico de Papiloma Escamoso Oral (AP.166795). A paciente foi encaminhada para o Serviço de Cirurgia de Cabeça e Pescoço (CEON/HUOC/UPE), entretanto, apesar ser informada do risco de transformação maligna, a paciente não prosseguiu com o tratamento, em virtude de problemas pessoais.

Retornou ao Serviço de Odontologia (CEON/HUOC/UPE), em novembro de 2019, e foi identificado expansão da lesão verrucosa em mucosa jugal direita e lábios (Figuras 2A e 2B), sendo realizada a terceira biópsia incisional, em 25/11/19, cujo laudo anatomopatológico (AP194048) e exame imunohistoquímico (A19-39286) apontaram Papiloma Escamoso Oral com focos de displasia epitelial de leve a moderada.

Figuras 2A e 2B. Aspecto clínico de lesão papilomatosa em mucosa jugal direita, comissura bucal correspondente, face interna de lábios superior e externa do inferior. (2019).
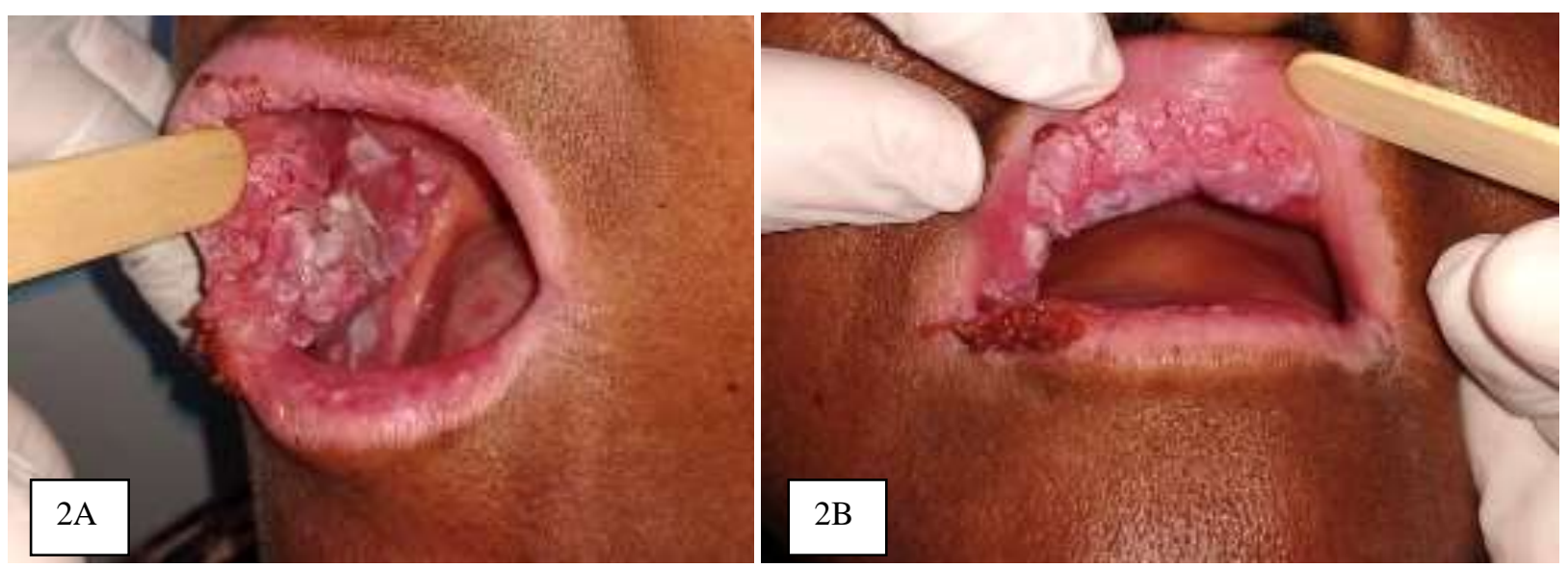

Fonte: Autores.

Foi agendado atendimento, novamente, com a equipe médica de Cirurgia de Cabeça e Pescoço (CEON/HUOC/UPE). No entanto, em 20/08/2020 (Figura 3), após reavaliação médica e odontológica, sendo confirmada a necessidade de tratamento cirúrgico agora com auxílio de reconstituição plástica, em virtude da progressão da lesão, a paciente mesmo esclarecida e incomodada com a proliferação do papiloma agora exteriorizado nos lábios, informou que não desejava realizar o procedimento cirúrgico, alegando problemas sociais, medo e ausência de apoio familiar. Foi encaminhada para o serviço social, de psicologia e psiquiatria, para que a auxiliassem no processo de entendimento da doença e melhor tomada de decisão; entretanto a paciente sumiu novamente. 
Figura 3. Aspecto clínico de lesão papilomatosa em mucosa jugal direita, comissura bucal correspondente e lábio inferior, já com displasia epitelial moderada (2020).

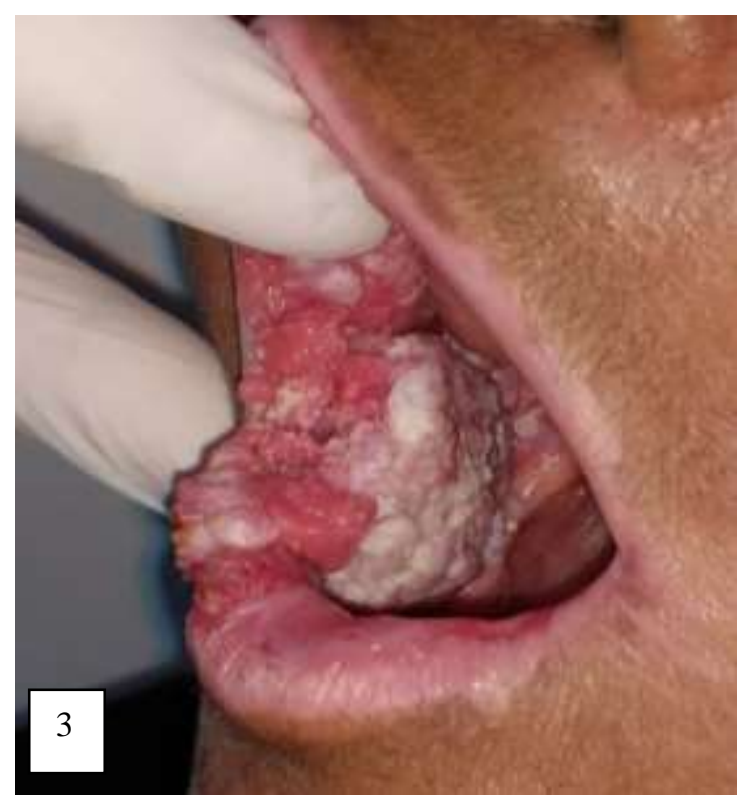

Fonte: Autores.

Em 12/01/2021 (Figuras 4A e 4B) a paciente buscou atendimento em uma Unidade de Pronto Atendimento (UPA), em decorrência da progressão acelerada da lesão, onde foi acolhida por uma equipe de assistência social, mas manteve a recusa frente ao tratamento cirúrgico indicado e não compareceu a consultas posteriores. As equipes médica e odontológica seguem no aguardo da paciente.

Figuras 4A e 4B. Lesão papilomatosa bastante extensa, já exteriorizada, em mucosa jugal direita, comissura bucal e lábios (2021).
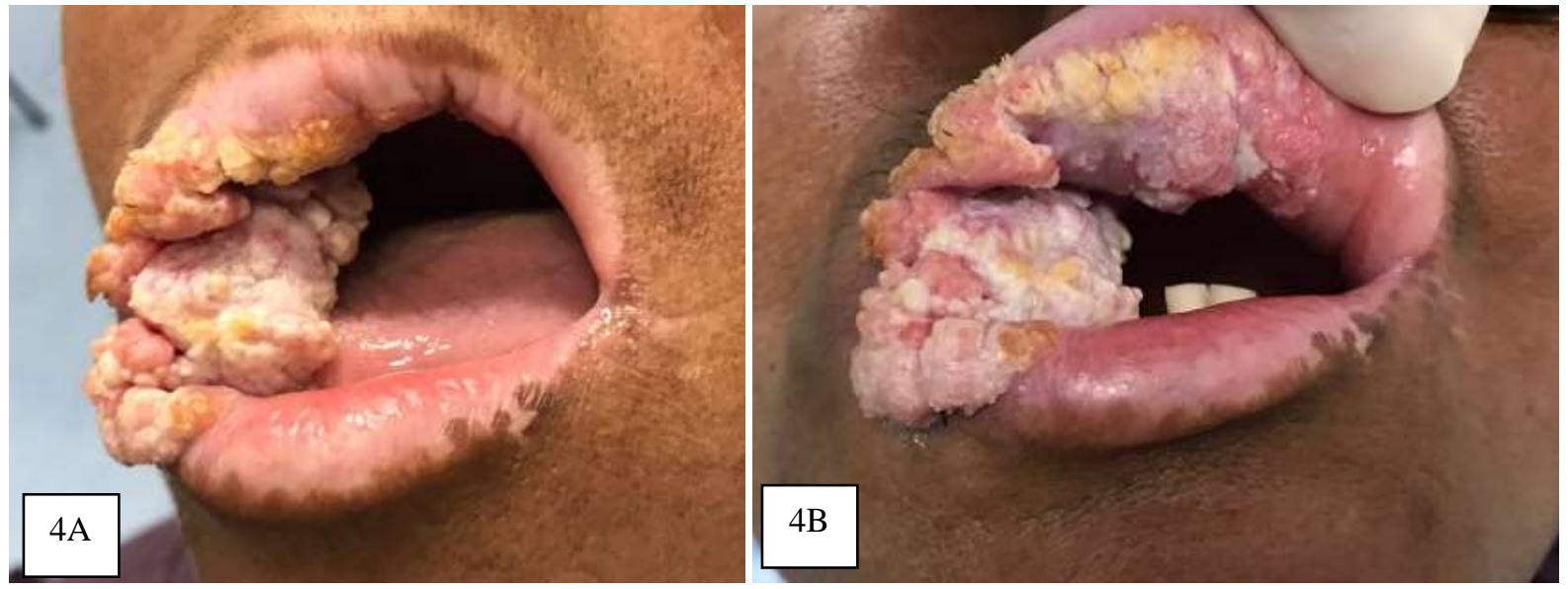

Fonte: Autores.

\section{Discussão}

O caso ora relatado corrobora com a literatura, pois o papiloma escamoso oral é a quarta lesão de mucosa mais comum, acometendo $1 \mathrm{em}$ cada 250 adultos e constitui aproximadamente $3 \%$ de todas as lesões orais submetidas à biópsia (Singh et al., 2016; Neville et al., 2016). Segundo Ferraro et al. (2011) e Tonioli et al. (2017) é uma manifestação mais comum 
em indivíduos entre 30 a 50 anos de idade. Carneiro et al. (2009) aponta que as lesões acometem principalmente o sexo feminino em 75\% dos casos, e Martins Filho et al (2009), em sua pesquisa estudou 83 casos de papiloma e verificou que as mulheres foram mais afetadas do que os homens numa proporção de 3:1 e a maioria dos casos ocorreu na $4^{\mathrm{a}}$ e $5^{\mathrm{a}}$ décadas de vida.

Clinicamente, os papilomas orais apresentam-se mais frequentemente com crescimento exofítico, isolado, peduncular, assintomático, com tamanho menor do que $1 \mathrm{~cm}$, cuja superfície mostra projeções digitiformes ou verrucosas, assemelhando-se a uma couve-flor, e coloração variando do rosa ao branco pálido, dependendo do grau de queratinização associado (Andrade et al., 2019; Nogueira et al., 2020). O tempo de evolução é variável, pode ser encontrado com início em poucas semanas até 20 anos (Carneiro et al., 2009). No caso relatado, o tempo de evolução é incerto, uma vez que a paciente é acompanhada desde 2016 e já apresentava lesão de tamanho significativo, sem saber informar sobre o início.

Quanto ao tamanho, as lesões raramente medem mais que $1 \mathrm{~cm}$, e segundo Neville et al. (2016) essas lesões crescem rapidamente até o tamanho máximo de cerca $0,5 \mathrm{~cm}$, contudo, lesões mais desenvolvidas atingindo $3,0 \mathrm{~cm}$ em seu maior diâmetro têm sido descritas. Nogueira et al. (2020), em seu trabalho, relatam um caso clínico de uma paciente com papiloma que envolvia praticamente toda a porção direita da língua e estendia-se do ápice lingual à região posterior, próximo às papilas valadas, com aproximadamente $6 \mathrm{~cm}$ no seu maior comprimento. Lesões de tal comprimento são escassas na literatura, por isso esse relato foi o que mais se aproximou do caso descrito, onde diagnosticou-se lesão com dimensões bastante extensas.

Os sítios preferencialmente acometidos incluem dorso de língua, palato mole e lábios, contudo, qualquer superfície oral pode ser afetada (Neville et al., 2016; Andrade et al., 2019; Nogueira et al., 2020). Martins Filho et al. (2009), verificaram que a mucosa jugal estava em quarto lugar dos locais de maior envolvimento, tal qual o caso clínico relatado.

Ainda no trabalho de Martins Filho et al. (2009), a displasia epitelial foi encontrada em apenas 2 casos dos 83 analisados, sendo ambas classificadas como moderadas. Semelhante ao trabalho de Martínez et al. (2016), que realizaram um estudo com o resultado histopatológico de 389 pacientes com biópsia de mucosa oral ou orofaríngea, onde encontraram um total de 27 casos $(6,8 \%)$ de displasia epitelial moderada e observaram que essas lesões são encontradas principalmente em pacientes com idade superior a 50 anos. Assim, observa-se que a transformação do epitélio em displasia epitelial moderada, como segue relatado neste caso, apresenta porcentagem baixa, entretanto ocorre.

A vulnerabilidade social da paciente contribuiu para a recusa ao tratamento cirúrgico indicado desde a primeira biopsia, cujo laudo anatomopatológico foi conclusivo para o papiloma escamoso oral. Compreendendo que as ações e serviços de saúde devem contemplar a autonomia e a corresponsabilização dos usuários, e respeitando a Lei no 10.406 do Código Civil (2002) que positivou em seu artigo $15^{\circ}$, no capítulo dos Direitos de Personalidade, que "ninguém pode ser constrangido a submeter-se, com risco de vida, a tratamento médico ou a intervenção cirúrgica”, cabe aos profissionais de saúde, entender que a recusa da paciente de iniciar ou manter determinadas terapias, guarda motivos e valores pessoais e desrespeitá-los poderia significar danos de maior gravidade que a própria enfermidade (Dadalto \& Pimentel, 2018).

Assim, o direito à recusa de tratamento é reconhecido pelo Código Civil brasileiro como um dos direitos da personalidade, ou seja, um direito garantido para toda e qualquer pessoa, em razão de sua simples condição de ser pessoa humana (Berti, 2000). Apesar de ser angustiante ver a progressão da lesão e a possibilidade de transformação maligna, sem a permissão para intervir, é importante continuar com o suporte e disponibilidade para atendimento, frisando sempre a possibilidade de tratamento ideal a fim de favorecer a melhor tomada de decisão por parte da paciente.

A atenção à pessoa humana requer o reconhecimento de sua dignidade. É necessário que o indivíduo seja percebido como ser único nas suas dimensões: biológica, psicológica, social e espiritual. Deste modo, a bioética é essencial na formação e atuação do profissional de saúde a fim de que possa realizar uma abordagem integral do processo saúde-doença e que, 
consequentemente, promova o respeito à dignidade da pessoa e resolução dos dilemas morais advindos do campo da assistência em saúde.

A comparação entre a bioética e a deontologia/ética profissional evidencia a proximidade de alguns de seus objetos de estudo. Ambas lidam com a ética, a moral, valores, condutas e as relações humanas em geral. Entretanto, diferem na maneira como abordam os diferentes conflitos. Enquanto a deontologia utiliza- se de códigos, prescrições e respostas determinadas, lançando mão muitas vezes de mecanismos punitivos àqueles profissionais que não se adequam às regras, direcionando, portanto, ao legalismo, a bioética, em contrapartida, refere-se à legitimação das decisões morais, atuando por meio da aplicação de uma ética minimalista para mediar os conflitos, não tendo por obrigatoriedade resolvê-los. Deve sim utilizá-los como oportunidade para reflexão e desenvolvimento da capacidade humana de respeitar as moralidades diversas e com elas conviver pacificamente, respeitando os princípios da beneficência/ não maleficência, autonomia e justiça (Garrafa, 2003; Pyrrho et al., 2009).

Todos esses princípios devem ser considerados "ferramentas" de trabalho e, diante de um processo de decisão, é preciso lembrar do reconhecimento do valor da pessoa; em seguida, deve-se buscar fazer o bem para aquela pessoa (e evitar um mal); e respeitar suas escolhas (autonomia); e, por fim, deve-se ser justo. A Bioética é inerente ao exercício da boa prática médica/odontológica e pode proporcionar uma contribuição significativa ao Código de Ética Profissional.

O consentimento informado incorporado à prática profissional, favorece a relação entre o conhecimento científico e o conhecimento humanístico, pois o paternalismo, cartesianismo, ênfase na doença, individualismo, hedonismo e utilitarismo existem, mas devem ser combatidos em prol do reconhecimento e valorização da dignidade da pessoa humana, como um ser único e que deve ser considerado em sua totalidade - aspectos físicos, psicológicos, sociais e espirituais - tendo em vista a beneficência/não maleficência, autonomia e justiça, a fim de se conseguir agir eticamente diante de um conflito ético.

\section{Considerações Finais}

Diante da recusa da paciente em realizar o tratamento proposto, ainda é importante que seja realizado o acompanhamento do papiloma escamoso oral extenso com displasia epitelial moderada, tendo em vista a possibilidade de transformação maligna da lesão. Respeitar a autonomia do paciente e as condições psicossociais por ela relatadas, como a falta de apoio familiar, também são características de um sistema de saúde humanizado e constitui um dos principais desafios enfrentados pelos profissionais de saúde. Por fim, conhecer os limites da lei, os princípios éticos e bioéticos são imprescindíveis para que direitos, como a autonomia sejam garantidos a todos os cidadãos. Mais estudos são necessários para melhor entendimento e prática da bioética, a qual permeia o atendimento médico-odontológico, a fim de favorecer o diagnóstico, o tratamento e melhor prognóstico, respeitadas as individualidades e particularidades dos pacientes e prestado o apoio através das redes integradas de atenção à saúde.

\section{Referências}

Albrecht, J., Meves, A. \& Bigby, M. (2005). Case reports and case series from Lancet had significant impact on medical literature. J Clin Epidemiol, 58, 12271232 .

Andrade, S. A., Pratavieira, S., Paes, J. F., Ribeiro, M. M., Bagnato, V. S. \& Varotti, F. P. (2019). Papiloma escamoso oral: uma visão sob aspectos clínicos, de fluorescência e histopatológicos. einstein (São Paulo), 17(2), eRC4624. http://dx.doi.org/ 10.31744/einstein_journal/2019RC4624

Bardin, L. (2010). Análise de conteúdo. Edições 70.

Beauchamp, T. L. \& Childress, J. F. (2002). Principios de ética biomédica. (4a ed.), edições Loyola.

Berti, S. M. (2000). Fragilização dos Direitos da Personalidade. Revista da FMD, 3(5 e 6), 238-247.

Bourget, M. M. M. (2006). Programa Saúde da Família. Martinari, 168p.

Brasil (2004). Ministério da Saúde. Diretrizes da Política Nacional de Saúde Bucal. 
Brasil (2008). Ministério da Saúde. Série A. Normas e Manuais Técnicos. Cadernos de Atenção Básica. 17.

Cardoso, E. M. M. (2012). Aspectos históricos, fisiopatológicos e preventivos da infecção por Papiloma Vírus Humano - HPV. Universidade Federal de Minas Gerais [TCC]. Araçuai,

Carneiro, T. E., Marinho, A. S., Verli, F. D., Mesquita, A. T. M., Lima, N. L. \& Miranda, J. L. (2009). Oral squamous papilloma: clinical, histologic and immunohistochemical analyses. J Oral Sci, 51(3), 367-372.

Carvalho Fortes, P. A. \& Pavone Zoboli, E. L. C. (2009). Bioética e Saúde Pública: entre o individual e o coletivo. Bioética e Saúde Pública. 3.ed. São Paulo: Loyola.

Castro, T. M. P. G., Neto, C. E. R., Scala, K. A. \& Scala, W. A. (2004). Manifestações orais associada ao papilomavírus humano (hpv) conceitos atuais: revisão bibliográfica. Rev. Bras. Otorrinolaringol, 70(4), 546-550.

Código Civil - Lei 10406/02 | Lei nº 10.406, de 10 de janeiro de 2002.

Conselho Federal de Odontologia - CFO. Código de Ética Odontológico, aprovado pela Resolução CFO-118/2012.

Dadalto, L. \& Pimentel, W. (2018). Direito à recusa de tratamento: Análise da Sentença proferida nos autos nº 201700242266 - TJGO. RBDCivil, Belo Horizonte, 15, 159-175.

Estrela, C. (2018). Metodologia Científica: Ciência, Ensino, Pesquisa. (3a ed.), Editora Artes Médicas.

Feller, L., Khammissa, R. A., Wood, N. H. \& Lemmer, J. (2009). Epithelial maturation and molecular biology of oral HPV. Infect Agent Cancer, 4, p.16.

Ferraro, C. T. L., Canedo, N. H. S., Oliveira, S. P., Carvalho, M. G. C. \& Dias, E. P. (2011). Infecção oral pelo HPV e lesões epiteliais proliferativas associadas. J Bras Patol Med Lab, 47(4), 451-459.

Garrafa, V. (1995). Dimensão da Ética em Saúde Pública. São Paulo: Faculdade de Saúde Pública USP / Kellogg Foundation, p. 9.

Garrafa, V. (2003). Bioética e odontologia. In: Kriger L, organizador. Aboprev. Promoção de saúde bucal, paradigma e humanização. $3^{a}$ ed. São Paulo: Artes Médicas, 495-504.

Grm, H. S., Bergant, M. \& Banks, L. (2009). Human papillomavirus infection, cancer \& therapy. Indian J Med Res, 130(3), 277-285.

Guimarães, R. (2013). Dilemas morais e práticas de Saúde. Rev Saúde Pública, 47(2), 425-429.

Koche, J. C. (2011). Fundamentos de metodologia científica. Vozes.

Kottow, M. (1995). Introducción a la bioética. Santiago: Universitária.

Lakatos, E. M. \& Marconi, M. A. (2003). Fundamentos de metodologia científica. (5a ed.), Atlas.

Martínez, C., Hernández, M., Martínez, B. \& Adorno, D. (2016). Frecuencia de displasia epitelial y carcinoma escamoso en mucosa oral y orofaríngea en Chile, entre los años 1990 y 2009. Rev. méd. Chile, 144(2).

Martins Filho, P. R. S., Piva, M. R., Santos, S. T., Andrade, E. S. S. \& Silva, L. C. F. (2009). Papilomas de células escamosas da cavidade oral. Rev. Cir. Traumatol. Buco-Maxilo-fac., 9(3), 69-78

Montenegro, L. A. S., Veloso, H. H. P. \& Cunha, P. A. S. M. A. (2014). Papiloma vírus humano como fator carcinogênico e co-carcinogenico do câncer oral e da orofaringe. Rev Odontol Bras Central, 23(67), 217-225.

Neville, B. W., Damm, D., Allen, C. M. \& Bouquot J. E. (2016). Patologia epitelial. In: Patologia oral \& maxilofacial. (4a ed.), Elsevier Saunders.

Nickel, D. A., Lima, F. G. \& Silva, B. B. (2008). Modelos assistenciais em saúde bucal no Brasil. Cad. Saúde Pública, 24(2), 241-246.

Nogueira, E. F., Lopes, P. H., Souza, B. L., Bezerra, C. B., Vasconcellos, R. J. \& Torres, B. C. (2020). Partial glossectomy for treating extensive oral squamous cell papilloma. Braz J Otorhinolaryngol., 86, 654-657

Paulino, I., Bedin, L. P. \& Paulino, L. V. (2009). Estratégia Saúde da Família. Ícone, 448p.

Pereira, A. S., Shitsuka, D. M., Pereira, F. J. \& Shitsuka, R. (2018). Metodologia da pesquisa científica. UFSM.

Pereira, K. M. A., Santos, P. P. A., Rocha, D. A. P. \& Lima, K. C. (2007). Papilomavírus humano e câncer oral: uma revisão dos conceitos atuais. Rev. odontol. UNESP, 36(2), 151-156.

Porensky, E. K. \& Carpenter, B. D. (2016). Breaking bad news: Effects of forecasting diagnosis and framing prognosis. Patient Educ Couns, 99(1), 68-76.

Pyrrho, M., Prado, M. M., Cordón, J. \& Garrafa, V. (2009). Análise do Código de Ética Odontológica Brasileiro. Ciência \& Saúde Coletiva, 14(5), 19111918.

Ramos, D. L. P. \& Junqueira, C. R. (2007). Bioética: conceito, contexto cultural, fundamento e princípios. Bioética \& ética profissional. Guanabara Koogan.

Ramos, D. L. P. (2009). Bioética: pessoa e vida. São Caetano do Sul: Difusão, 374p.

Schramm, F. R. (2001). Bioética e Comunicação em Oncologia. $R B C$, 47(1), 25-32. 
Research, Society and Development, v. 10, n. 5, e44010515183, 2021

(CC BY 4.0) | ISSN 2525-3409 | DOI: http://dx.doi.org/10.33448/rsd-v10i5.15183

Silva, E. J., Corrêa, M. M. J., Santos, M. A. T. \& Flores, L. S. (2016). Considerações relacionadas ao diagnóstico e tratamen to do Papilomavírus Humano (HPV) em cavidade oral. Rev. Odontol. Univ. Cid. São Paulo, 28(2), 117-125.

Simonato, L. \& Miyahara, G. (2007). O papel do papilomavírus humano na carcinogênese bucal. Rev Bras Cancerol, 53(4), 471-476.

Singer, P. (1994). Ética Prática. Martins Fontes, 399p.

Singh, A. K., Malik, U., Malhotra, S. \& Kumar, A. (2016). Squamous papilloma: A report of two cases with review of literature. J Indian Acad Oral Med Radiol, 28(1), 102-104.

Tonioli, I. B., Tomo, S., Ferreira, A. S. \& Simonato, L. E. (2017). Diferentes aspectos clínicos do papiloma escamoso bucal. Proceedings of the IX Jornada Odontológica da Universidade Brasil -2017/Annual Meeting. Arch Health Invest, p.6.

Vidal, A. K. L., Caldas Júnior, A. F., Mello, R. J. V. \& Abreu-e-Lima, M. C. C. (2006). Papiloma Vírus Humano (HPV) como fator de risco para o carcinoma escamoso celular (CEC): revisão de literatura. Odontol. Clín-cient, 5(1), 7-25.

Vidal, A. K. L., Caldas Júnior, A. F., Mello, R. J. V., Brandão, V. R. A., Rocha, G. I. \& Taromaru, E. (2004). HPV detection in oral carcinomas. J Bras Patol Med Lab, 40(1), 21-26.

World Health Organization - WHO (2001). Geneva.

Yin, R. K. (2015). Estudo de caso: planejamento e métodos. (5a ed.), Bookman. 